\title{
Two-Dimensional Phonon Polariton Heat
}

\section{Transport}

Laurent Tranchant, ${ }^{* \dagger}$ Satoki Hamamura, ${ }^{\dagger}$ Jose Ordonez-Miranda, ${ }^{\ddagger}$ Tomohide

Yabuki, ${ }^{\dagger}$ Alejandro Vega-Flick, " Fernando Cervantes-Alvarez, "Juan Jose

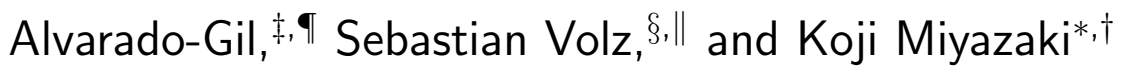

$\dagger$ Department of Mechanical and Control Engineering, Kyushu Institute of Technology, 1-1

Sensui-cho, Tobata-ku, Kitakyushu 804-8550, Japan

$\ddagger$ Institut Pprime, CNRS, Université de Poitiers, ISAE-ENSMA, F-86962 Futuroscope,

Chasseneuil, France

\Applied Physics Department, CINVESTAV-Unidad Mérida, Carretera Antigua a Progreso km 6, Cordemex, Mérida, Yucatán, 97310, Mexico

$\S L I M M S / C N R S-I I S(U M I 2820)$, Institute of Industrial Science, University of Tokyo, 4-6-1

Komaba, Meguro-ku, Tokyo 153-8505 Japan

||Laboratoire d'Energétique Moléculaire et Macroscopique, Combustion, UPR CNRS 288,

CentraleSupélec, Université Paris-Saclay, Bat. Eiffel, 3, rue Joliot Curie, 91192

Gif-sur-Yvette cedex - France

E-mail: tranchant.laurent191@mail.kyutech.jp; miyazaki.koji055@mail.kyutech.jp

This appendix to the letter gives additional information about the theoretical background, the experimental setup, the experimental procedure and complementary data, which support the discussion presented in our letter. This part has been written to add some details we think necessary to properly understand our demonstration. If the reader cannot find some information, we invite him/her to contact us directly. 
This PDF file contains methods and supplementary figures S1-S16.

\section{Theoretical analysis of the SPhP thermal conductivity}

\section{Broadening and spatial confinement of surface waves}

The well-known dispersion relation of SPhPs along a plane interface is:

$$
\beta=\frac{\omega}{c} \sqrt{\frac{\varepsilon_{\mathrm{SiO}_{2}}}{1+\varepsilon_{\mathrm{SiO}_{2}}}},
$$

while, the one for a free-standing $\mathrm{SiO}_{2}$ thin film is: ${ }^{1}$

$$
\begin{gathered}
\beta_{R}=\frac{\omega}{c}\left[1+\frac{\left(\frac{\omega}{c} t\right)^{2}}{8}\left(1-\frac{2 \mathrm{Re}\left(\varepsilon_{\mathrm{SiO}_{2}}\right)}{\left|\varepsilon_{\mathrm{SiO}_{2}}\right|^{2}}+\frac{\left(\operatorname{Re}\left(\varepsilon_{\mathrm{SiO}_{2}}\right)^{2}-\operatorname{Im}\left(\varepsilon_{\mathrm{SiO}_{2}}\right)^{2}\right)}{\left|\varepsilon_{\mathrm{SiO}_{2}}\right|^{4}}\right)\right], \\
\beta_{I}=\frac{\omega}{c} \frac{\left(\frac{\omega}{c} t\right)^{2}}{8} \frac{\operatorname{Im}\left(\varepsilon_{\mathrm{SiO}_{2}}\right)}{\left|\varepsilon_{\mathrm{SiO}_{2}}\right|^{2}}\left(1-\frac{\operatorname{Re}\left(\varepsilon_{\mathrm{SiO}_{2}}\right)}{\left|\varepsilon_{\mathrm{SiO}_{2}}\right|^{2}}\right),
\end{gathered}
$$

where $c$ is the light speed in vacuum and $t$ is the thickness of the film. Equations (2) and (3) apply for very thin freestanding films (with a thickness smaller than $300 \mathrm{~nm}$ ). ${ }^{1}$ Because the dielectric constant of glass is complex, we consider that the wave vector $\beta$ is complex $\left(\beta=\beta_{R}+i \beta_{I}\right)$ and the frequency $\omega$ is real, which is a common approach to describe the SPhP propagation. ${ }^{1,2}$

The dispersion relations (1) and (2) are presented in Figure S1a, where the frequency $\omega$ is plotted as a function of the real part of the wave vector $\beta_{R}$. We can see that the dispersion relation of SPhPs for a plane interface (solid blue line) crosses the light line (black dashed one) for frequencies following the conditions of existence of SPhPs (red area), whereas the dispersion relation of $\mathrm{SPhPs}$ for a $100 \mathrm{~nm}$ thick film (solid red line) follows closely the light 
line and is located on its right side for all frequencies. The light line usually defines the radiative modes (whose wave vectors are smaller than the light line) from the evanescent modes (whose wave vectors are larger than the light line). This trend is confirmed by Figure $\mathrm{S} 1 \mathrm{~b}$, which shows the frequency as a function of the confinement ratio $\delta$ (ratio between the transverse attenuation length and the transverse wavelength). For $\delta<<1$ the modes are strongly spatially confined along the surface, while for $\delta>>1$ the modes tend to propagate

in the far field and we cannot call them surface modes. In Figure S1b, the ratio $\delta$ takes values smaller than 1 , in the range of frequency of $\mathrm{SPhP}$ (red area) for the plane interface. However, in the case of the thin film, this ratio is smaller than 1 on a much broader range of frequency, especially for low frequency. As a result, the existence of surface waves for a glass plane interface, is limited to the $\mathrm{SPhP}$ range of frequency, whereas for thin films it is not limited any more to this narrow range of frequency. Following a previous study, ${ }^{3}$ we call the additional surface modes, Zenneck modes.

Figure S1c presents the spectrum of the surface waves propagation length, which shows that Zenneck modes have the highest propagation length over a very broad range of frequency, whereas SPhPs have the shortest ones over a narrow spectrum. As a result, we believe that the Zenneck modes are the main contributor to the enhancement of the heat transfer of amorphous silica thin films.

\section{Thermal conductivity due to surface waves}

We compute the in-plane heat flux using the kinetic theory and following a similar demonstration as the one reported by Chen et al. ${ }^{2}$ A scheme of the suspended membrane is presented in the Figure S2. We use the diffusion approximation even if the size of the membranes are much smaller than the intrinsic propagation length of surface waves, because only the diffusive part of the surface waves will contribute to the thermal conductivity. The heat flux $Q_{y}$ of surface waves in the y-direction (direction where we assume most of the energy is 
(a)

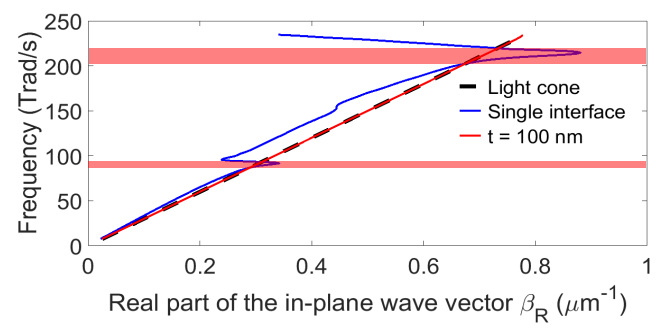

(b)

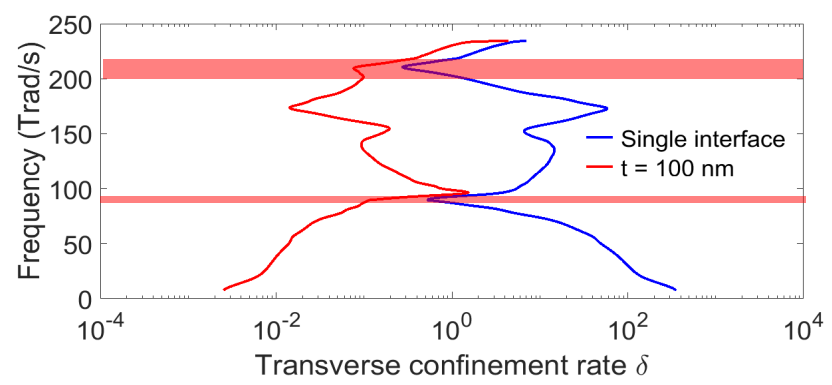

(c)

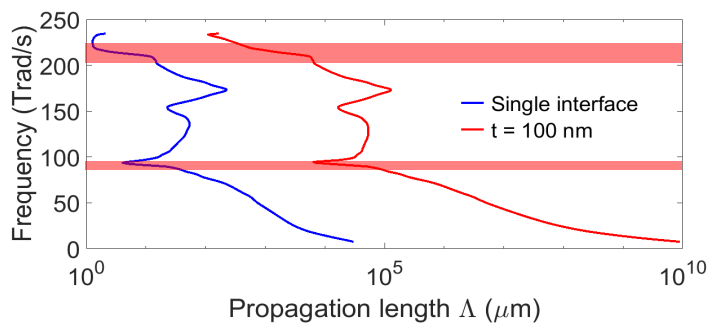

(d)

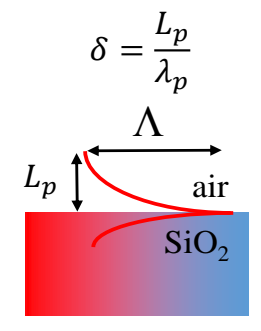

Supplementary Figure S1: (a) Dispersion relation, (b), transverse confinement rate, and (c), propagation length for an amorphous silica plane surface and a $100 \mathrm{~nm}$ thick suspended amorphous silica thin film as a function of frequency. The red areas correspond to the $\mathrm{SPhP}$ range of frequency for a lossless material $\left(\operatorname{Re}\left(\varepsilon_{\mathrm{SiO}_{2}}(\omega)\right)<-1\right)$. (d) Scheme explaining the physical meaning of the transverse confinement rate $\delta$. It is the ratio between the transverse attenuation length of the surface waves $L_{p}$ in the air over its wavelength in the transverse direction also in the air $\lambda_{p}=\frac{1}{\operatorname{Im}\left(p_{\text {air }}\right)}$ with $p_{\text {air }}$ the transverse wave vector of the surface electromagnetic wave in the $\operatorname{air}\left(p_{\text {air }}^{2}=\beta^{2}-(\omega / c)^{2}\right){ }^{1}$ 
transferred) is calculated considering that they are propagating as a bidimensional gas:

$$
Q_{y}=\frac{1}{a b t} \sum_{k_{x}, k_{y}} \hbar \omega f \vec{v} \cdot \vec{y}
$$

where $v$ is the group velocity of the surface waves and $f$ is their distribution function, which is determined by the Boltzmann transport equation under the relaxation time approximation with steady-state conditions and no external force, as follows:

$$
v_{x} \frac{\partial f}{\partial x}+v_{y} \frac{\partial f}{\partial y}=-\frac{f-f^{0}}{\tau}
$$

with $f^{0}$ being the Bose-Einstein equilibrium distribution function and $\tau$ the relaxation time. In this equation the distribution function is supposed to vary only in the $\mathrm{x}$ and $\mathrm{y}$ directions, because the temperature varies only in these directions. Based on the diffusion approximation, we can make the following simplifications: $\frac{\partial f}{\partial x} \approx \frac{\partial f^{0}}{\partial x}$ and $\frac{\partial f}{\partial y} \approx \frac{\partial f^{0}}{\partial y}$, which reduce equation (5) to:

$$
f=f^{0}-v_{x} \tau \frac{\partial f^{0}}{\partial x}-v_{y} \tau \frac{\partial f^{0}}{\partial y}=f^{0}-\Lambda \cos (\varphi) \frac{\partial f^{0}}{\partial x}-\Lambda \sin (\varphi) \frac{\partial f^{0}}{\partial y}
$$

where $\Lambda=\|\vec{v}\| \tau$ is the intrinsic propagation length of the surface waves.

We can now rewrite the heat flux in equation (4) as:

$$
Q_{y}=\frac{1}{a b t} \iint \hbar \omega v\left(f^{0}-\Lambda \cos (\varphi) \frac{\partial f^{0}}{\partial x}-\Lambda \sin (\varphi) \frac{\partial f^{0}}{\partial y}\right) \cos (\varphi) g_{2 D}\left(k_{/ /}\right) d^{2} k_{/ /}
$$

where we have used the notation in polar coordinates, with $k_{/ /}$being the in-plane component of the wave vector (see Figure S2) and $g_{2 D}\left(k_{/ /}\right)$the bidimensional density of states, such as $g_{2 D}\left(k_{/ /}\right) d^{2} k_{/ /}=\frac{a b}{4 \pi^{2}} d \varphi k_{/ /} d k_{/ /}$. The net in-plane surface waves heat flux $Q_{n e t}$ is then given 


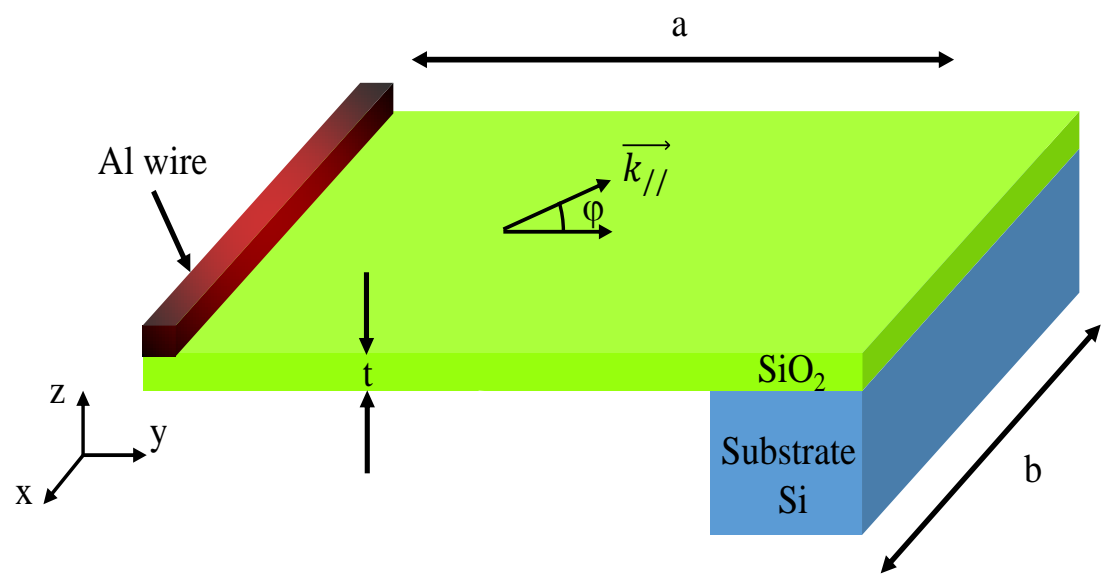

Supplementary Figure S2: Scheme of half of the suspended silica membrane. The surface electromagnetic waves propagate in the $\mathrm{x}-\mathrm{y}$ plane with a wave vector $k_{/ /}$, their propagation is constraint by the aluminum wire and the edges of the suspended area.

by:

$$
Q_{\text {net }}=Q_{y}\left(-\frac{\pi}{2} \leq \varphi \leq \frac{\pi}{2}\right)+Q_{y}\left(\frac{\pi}{2} \leq \varphi \leq \frac{3 \pi}{2}\right)
$$

which leads, after a few calculations, to the formula:

$$
Q_{\text {net }}=-\frac{1}{4 \pi t} \int \hbar \omega v \Lambda \frac{\partial f^{0}}{\partial x} k_{/ /} d k_{/ /}
$$

Equation (9) can be written as the Fourier's law $Q_{\text {net }}=-\kappa \partial T / \partial x$ with an effective thermal conductivity:

$$
\kappa=\frac{1}{4 \pi t} \int \hbar \omega v \Lambda \frac{\partial f^{0}}{\partial T} k_{/ /} d k_{/ /}
$$

Equation (10) represents the contribution of surface waves to the total thermal conductivity $\left(\kappa_{T}=\kappa+\kappa_{B}\right)$ of thin films. Taking into account that we experimentally measure $\kappa_{T}$, we have used $\kappa_{B}=1.4 \mathrm{~W} /(\mathrm{m} . \mathrm{K})$, the bulk thermal conductivity of amorphous silica, to properly compare our experimental measurements with the predictions of equation (10). 


\section{Thermal modelling of the $3 \omega$ experiment}

Here we detail the theoretical method to retrieve the thermal conductivity and the thermal diffusivity of the samples based on the previous studies made by Jain and Goodson. ${ }^{4}$ An aluminum wire deposited at the center of the suspended glass membranes will act as a heater and as a temperature sensor due to its temperature-dependent resistance. By applying a $\mathrm{DC}$ and then an $\mathrm{AC}$ voltage across the wire the thermal conductivity and diffusivity of the samples can be separately determined. The section considered is half of the membrane located between $y=0$ and $y=a, a$ being the half-width of the membrane and $b$ its total length (Figure S2). If only a DC voltage is applied to the wire, the heat conduction equation in the membrane will be:

$$
\frac{\partial^{2} \theta}{\partial x^{2}}+\frac{\partial^{2} \theta}{\partial y^{2}}=0
$$

with $\theta$ being the steady-state temperature rise of the silicon dioxide membrane. There is no heat generation inside the silicon dioxide membrane and the temperature gradient in the $\mathrm{z}$ direction is neglected because of the very small thickness of the membrane (lower than 300 $\mathrm{nm}$ in the experiments). As a result the heat propagates mainly in the xy plane, thus $\kappa$ is the membrane in-plane thermal conductivity. The equation 11 can be easily solved using the following boundary conditions:

$$
\theta(0, y)=\theta(b, y)=\theta(x, a)=0 \text { and }\left.\kappa \frac{\partial \theta}{\partial y}\right|_{y=0}=-\frac{R I^{2}}{2 b t}
$$

with $R$ the resistance of the metal wire and $I$ the electrical current. These conditions suppose that the boundaries of the membrane, in contact with the substrate, are isothermal and that half of the heat flux coming from the metallic wire is directly transmitted to the halfmembrane without losses. The solution of this problem requires the separation of variables and has already been published. ${ }^{4}$ Here we present in our case the average steady-state metal 
heater temperature rise:

$$
\bar{\theta}_{D C}=\frac{4 R I^{2}}{\kappa t} \sum_{n=0}^{+\infty} \frac{\tanh \left((2 n+1) \frac{\pi a}{b}\right)}{[(2 n+1) \pi]^{3}}
$$

This equation is used to obtain the in-plane thermal conductivity of the membranes.

By applying an oscillatory voltage of frequency $\omega$, the temperature rise of the heater contains a steady-state and a transient component. We focus on the transient temperature rise of the membrane $\psi$, which is given by the following heat conduction equation:

$$
\frac{d^{2} \psi}{\partial x^{2}}+\frac{\partial \psi^{2}}{\partial y^{2}}=\frac{1}{\alpha} \frac{\partial \psi}{\partial t}
$$

where $\alpha$ is the thermal diffusivity of the membrane. The proper boundary conditions an be written as follows:

$$
\psi(0, y, t)=\psi(b, y, t)=0, \quad \psi(x, a, t)=\psi(x, y, 0)=0 \quad \text { and }\left.\quad \kappa \frac{\partial \psi}{\partial y}\right|_{y=0}=\frac{R I_{0}^{2} \cos (2 \omega t)}{4 b t}
$$

with $I_{0}$ being the amplitude of the oscillatory current. The solution of the equation (14) has already been proposed by Jain and Goodson ${ }^{4}$ but its formula is complicated and does not respect the boundary condition $\psi(x, y, 0)=0$. This has no effect on their final results because in practice measurements are done for a time long enough to prevent any artefact due to the condition $t=0$. However, we would like to report the correct solution respecting all the boundary conditions and whose final formula is easy to use for our experiments. We used a Laplace transform of equation (14) to obtain:

$$
\frac{d^{2} \Psi}{\partial x^{2}}+\frac{\partial \Psi^{2}}{\partial y^{2}}=\frac{s}{\alpha} \Psi
$$

where $\Psi$ is the Laplace transform of $\psi$ and $s$ the Laplace parameter. The separation of variables enables to solve equation (16) before using the inverse Laplace transform to obtain 
the solution for the transient thermal response:

$$
\begin{aligned}
\psi(x, y, t)= & \frac{R I_{0}^{2}}{2 \pi \kappa t b} \operatorname{Re}\left(\sum _ { n = 0 } ^ { + \infty } \frac { \operatorname { s i n } [ \frac { ( 2 n + 1 ) \pi x } { b } ] } { n + \frac { 1 } { 2 } } \left[\frac{\sinh \left[\Delta_{2 n+1}(a-y)\right] e^{2 i \omega t}}{\Delta_{2 n+1} \cosh \left(\Delta_{2 n+1} a\right)}\right.\right. \\
& \left.\left.-\frac{2 \alpha}{a} \sum_{m=0}^{\infty} \frac{(-1)^{m} \gamma_{n, m} e^{-\gamma_{n, m} t} \sin \left[\pi\left(m+\frac{1}{2}\right)\left(1-\frac{y}{a}\right)\right]}{\gamma_{n, m}^{2}+4 \omega^{2}}\right]\right)
\end{aligned}
$$

where

$$
\Delta_{2 n+1}=\sqrt{\left(\frac{(2 n+1) \pi}{b}\right)^{2}+\left(\frac{2 i \omega}{\alpha}\right)^{2}}
$$

and

$$
\gamma_{n, m}=\alpha \pi^{2}\left[\left(\frac{2 n+1}{b}\right)^{2}+\left(\frac{m+\frac{1}{2}}{a}\right)^{2}\right] .
$$

Finally the integration of equation (17) over the metal heater yields the theoretical expression of the measured amplitude of the transient temperature rise:

$$
\bar{\theta}_{A C}=\frac{2 R I_{0}^{2}}{\kappa t b}\left|\sum_{n=0}^{+\infty} \frac{\tanh \left(\Delta_{2 n+1} a\right)}{\Delta_{2 n+1}[(2 n+1) \pi]^{2}}\right| .
$$

This formula is used to determine the thermal diffusivity $\alpha$ of our samples.

\section{Study of the radiation losses}

For a film width larger than $500 \mu \mathrm{m}$, radiation losses at room temperature exceed $10 \%$ of the heat conduction inside the film and thus cannot be neglected. The thermal model has to be modified by including the radiation losses in the $\mathrm{AC}$ and $\mathrm{DC}$ problems. For the DC problem, the new boundary condition modifying the equation (12) is:

$$
-\left.\kappa \frac{\partial \theta}{\partial y}\right|_{y=0}+8 \epsilon \sigma T_{0}^{3} \theta(x, 0)=\frac{R I^{2}}{2 b t}
$$


with $\epsilon$ the emissivity of the silica film and $\sigma$ the Stefan-Boltzmann constant, the final result is:

$$
\bar{\theta}_{r a d-D C}=\frac{4 R I^{2}}{\kappa t} \sum_{n=0}^{+\infty} \frac{\tanh \left((2 n+1) \frac{\pi a}{b}\right)}{[(2 n+1) \pi]^{3}} \frac{1}{1+\frac{\gamma}{(2 n+1)} \tanh \left(\frac{(2 n+1) \pi a}{b}\right)}
$$

with $\gamma=\frac{\left(8 \epsilon \sigma T_{0}^{3} b\right)}{\pi \kappa}$. This parameter $\gamma$ is lower than $2 \times 10^{-3}$ for all the measured samples and thus we can assume that $\bar{\theta}_{\text {rad-DC }} \approx \bar{\theta}_{D C}$. The modification of the AC problem provides similar results, the new boundary condition modifying the equation (15) is:

$$
-\left.\kappa \frac{\partial \psi}{\partial y}\right|_{y=0}+8 \epsilon \sigma T_{w}^{3} \psi(x, 0, t)=-\frac{R I_{0}^{2} \cos (2 \omega t)}{4 b t}
$$

with $T_{w}$ the average temperature of the metal wire, in this context the Laplace solution of the equation (16) is:

$$
\Psi_{\text {rad }}(x, y, s)=-\frac{R I_{0}^{2}}{2 \pi \kappa t b} \frac{s}{s^{2}+4 \omega^{2}} \sum_{n=0}^{+\infty} \frac{\sin \left(\frac{(2 n+1) \pi x}{b}\right)}{(2 n+1) \lambda_{n}} \frac{\sinh \left(\frac{\pi}{b} \lambda_{n}(a-y)\right)}{\cosh \left(\frac{\pi a}{b} \lambda_{n}\right)} \frac{1}{1+\frac{\gamma_{\alpha}}{\lambda_{n}} \tanh \left(\frac{\pi a}{b} \lambda_{n}\right)}
$$

with $\lambda_{n}=\sqrt{(2 n+1)^{2}+\frac{s b^{2}}{\pi^{2} \alpha}}$ and $\gamma_{\alpha}=\frac{8 \epsilon \sigma T_{w}^{3} b}{\pi \kappa}$. Again the modification of the thermal problem depends on the parameter $\gamma_{\alpha}$ which takes values lower than $2 \times 10^{-3}$ in our measurements and can be neglected, thus $\Psi_{\text {rad }}(x, y, s) \approx \Psi(x, y, s)$ with $\Psi(x, y, s)$ the solution of the thermal problem without radiation losses. As a result we can use the thermal model neglecting the radiation losses to extract the thermal properties of our films at the condition of a limited temperature rise (around $20-25 \mathrm{~K}$ in our case) to enable the linearization of the radiative heat flux. 


\section{Dependence of the thermal conductivity on the half-}

\section{width}

The thermal conductivity dependence on the film half-width is displayed in Figure S3. For films thinner than $45 \mathrm{~nm}$, we can observe two effects: when the half-width is kept constant, the thermal conductivity $\kappa$ of thinner films is higher, while the $\kappa$ of films with a similar thickness (30-45 nm) dramatically drops, as the membrane becomes narrower. This trend is not so clear for films thicker than $50 \mathrm{~nm}$ as the propagation of surface electromagnetic waves decreases and is constraint by the size of the suspended films.

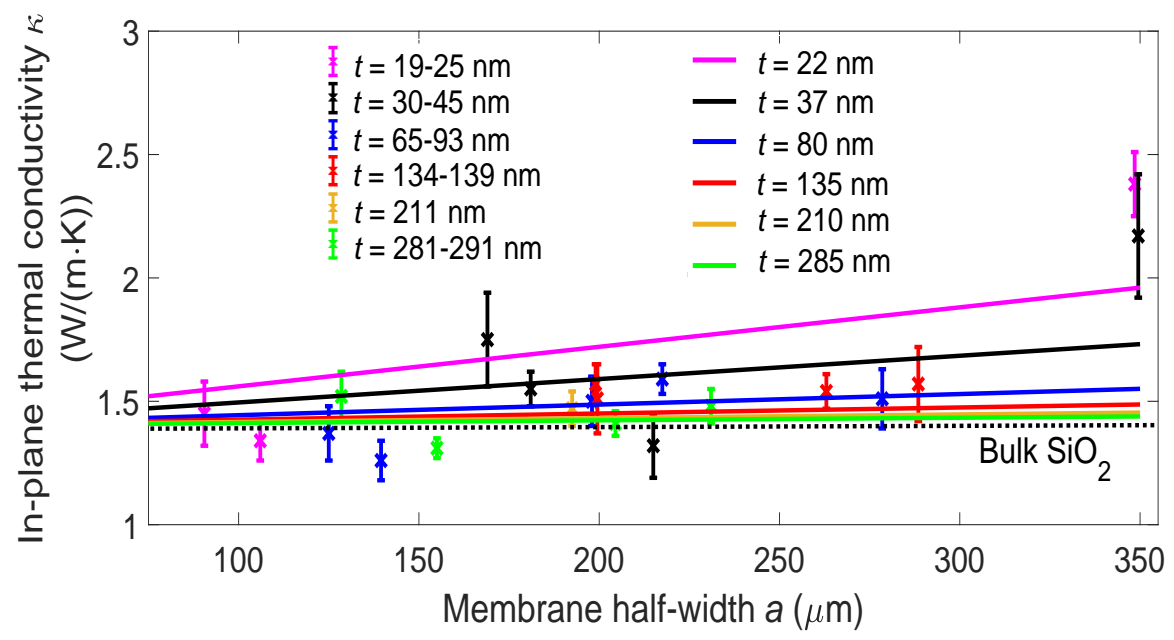

Supplementary Figure S3: Plot of the in-plane thermal conductivity as a function of the membrane half-width $a$. The experimental data are compared with the theoretical values predicted by model I, for different thicknesses.

\section{Fabrication of the samples}

The microfabrication process has been conducted at the center of Kitakyushu Foundation for the Advancement of Industry, Science and Technology (FAIS) and is summarized in the 
Figure S4. Silica is grown on 2 inch in diameter, $300 \mu \mathrm{m}$ thick, polished, p-type (100) silicon wafers by dry oxidation in a furnace at $1078{ }^{\circ} \mathrm{C}$, we use $\mathrm{NH}_{3}$ as a catalyst for thicknesses larger than $50 \mathrm{~nm}$ (step I). Then a $100 \mathrm{~nm}$ thick aluminum layer is sputtered on the samples followed by the deposition of the photoresist used for the patterning of the aluminum wire (steps II and III). The samples are exposed to UV rays before developping (step IV) and the non protected aluminum layer is etched by phosphoric acid (step V). Suspended silicon dioxide thin films use to crumple and break because of their inherent high compressive stress, ${ }^{5}$ in order to limit this effect we deposit a silicon nitride layer on it because it has a tensile stress and thus will counteract the compressive stress during the fabrication. This is why a $500 \mathrm{~nm}$ thick silicon nitride layer is deposited by PECVD (plasma-enhanced chemical vapor deposition) on the topside, on the backside a $1.2 \mu \mathrm{m}$ silicon nitride layer is also added for the patterning of the suspended membranes (step VI). Photoresist layer is again added, UV exposed, developed before the back etching of the silicon nitride layer by RIE (reactive ion ecthing) (steps VII-IX). At this time an additional RIE of a residual silicon dioxide layer on the backside is required, otherwise it will block the final wet etching of the silicon substrate. Then after removal of the photoresist, three layers of silicon dioxide and a layer of silicon nitride are added to protect the topside during the wet etching of the substrate (step X). The two $500 \mathrm{~nm}$ thick $\mathrm{SiO}_{2}$ layers are deposited by PECVD and thus are highly porous, the $250 \mathrm{~nm}$ thick water glass layer will fill the porosity of the lower layer and will strengthen the topside protection. The top PECVD silicon nitride layer will stabilize this protection and its thickness, $1.2 \mu \mathrm{m}$, in addition to the thicknesses of the three glass layers and the other topside silicon nitride layer will prevent any accidental top etching of the lowest $\mathrm{SiO}_{2}$ film. After that we proceed to the wet etching of the silicon substrate with an aqueous solution of TMAH (tetramethylammonium hydroxide) (20\%) (step XI) before the dry etching by RIE of the top protective layers (step XII). To avoid excessive etching the remaining thicknesses of the top protective layers are measured to estimate properly the etching time. Finally the thickness of the obtained samples is measured by spectroscopic reflectance at the edges of 
the suspended membranes. The thermal contact between the aluminium wire and the silicon dioxide film is not perfect and a thermal contact resistance may be important at this interface due to the wrinkles (on the order of $10^{-7}-10^{-8} \mathrm{~m}^{2} \cdot \mathrm{K} / \mathrm{W}^{6}$ ). However in our measurements the maximal heat flux transferred from the metal wire to the silica film is $Q_{\max } \approx 1.7 \times 10^{4}$ $\mathrm{W} / \mathrm{m}^{2}$ leading to a maximum temperature difference at the interface $\mathrm{Al} / \mathrm{SiO}_{2}$ around $10^{-3}$ $\mathrm{K}$, therefore the effect of thermal contact resistance is negligible in this study.

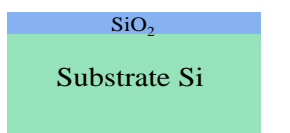

(1)
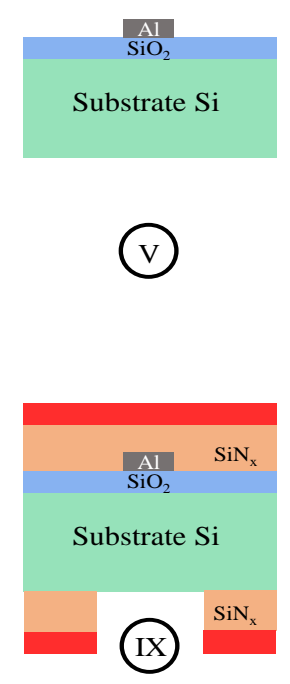

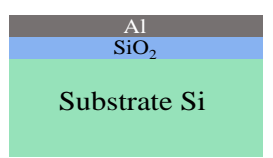

(11)
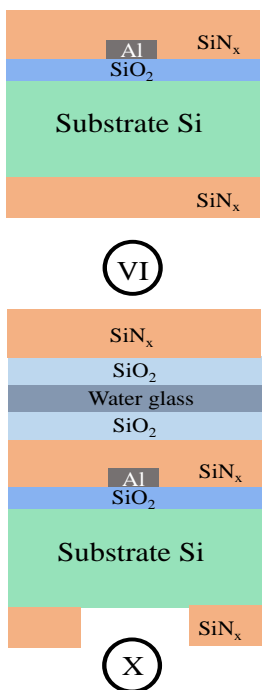

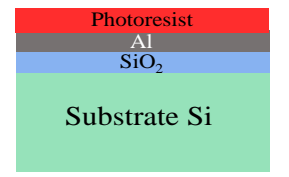

(III)

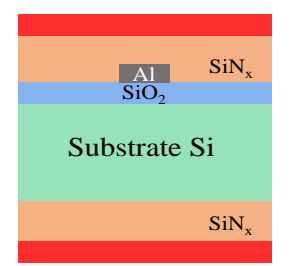

(VII)

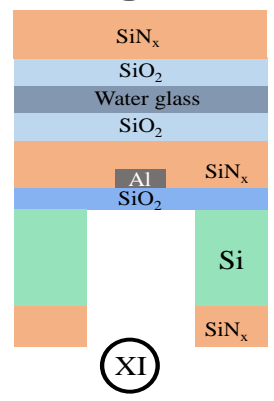

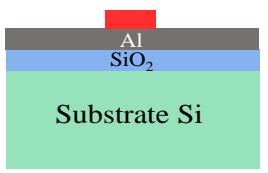

(IV)

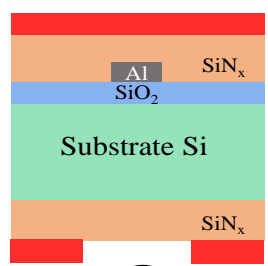

(VIII)

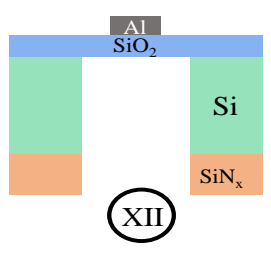

Supplementary Figure S4: Microfabrication process of freestanding $\mathrm{SiO}_{2}$ membranes by dry oxidation.

\section{Purity of samples}

The purity of the samples has been checked by two methods: indirectly by computing the ratio $\kappa / \alpha=\rho C$ from our results (Figure S5a) and directly by measuring the energy dispersive X-ray spectroscopy (EDS) spectrum of the samples (Figure S5b) with a scanning electron microscope (SEM, Hitachi, S-3400N) equipped with an EDAX Genesis-XM2 energy disper- 
sive X-ray spectroscope. The quantity $\rho C$ is also called the volumetric heat capacity and is independent of the thickness for these sizes because confinement effects start to appear for thicknesses lower than $10 \mathrm{~nm} .{ }^{7}$ We compared our measured volumetric heat capacity data with a referenced value for silica at room temperature ${ }^{8}$ and they are in fair agreement (Figure S5a) suggesting that the quality of our silica samples is generally good. Moreover the volumetric heat capacity remains constant for a decreasing thickness proving the physical consistency of the measured thermal properties enhancement. However the purity of the films is further investigated through EDS, the results vary among the samples and are presented in the Table S1 and an EDS spectrum is presented as an illustration in the Figure S5b. The $137 \mathrm{~nm}$ and $200 \mathrm{~nm}$ thick samples show an excess of silicon regarding to oxygen which might be due to a non complete thermal oxidation of silicon at the early stage of the microfabrication process. However the thinnest samples show atomic percentages close to the stoechiometric proportions of $\mathrm{SiO}_{2}$ and even an excess of oxygen regarding to silicon for the $20 \mathrm{~nm}$ thick film. This excess can be due to additional hydroxide which cannot be detected by EDS. These different impurities are a possible explanation of the discrepancies in the Figure 3 (in the letter) between experimental and theoretical data.

Table S1: Atomic percentages of silica suspended films measured by X-ray EDS.

\begin{tabular}{|c|c|c|c|c|c|}
\hline Film thickness (nm) & 20 & 30 & 37 & 137 & 200 \\
\hline \multirow{2}{*}{ Atomic percentage } & $77 \% \mathrm{O}$ & $65 \% \mathrm{O}$ & $68 \% \mathrm{O}$ & $59 \% \mathrm{O}$ & $61 \% \mathrm{O}$ \\
& $23 \% \mathrm{Si}$ & $35 \% \mathrm{Si}$ & $32 \% \mathrm{Si}$ & $41 \% \mathrm{Si}$ & $39 \% \mathrm{Si}$ \\
\hline
\end{tabular}

\section{Thermal properties measurement}

First of all the temperature coefficient of resistance (TCR) of the aluminium heater needs to be calibrated, in order to do that we measured the resistance of the Al wires placed in an electric furnace. We controlled the temperature of the samples with a thermocouple pasted on the surface of the wafer and waited for the thermal equilibrium of the whole 
(a)
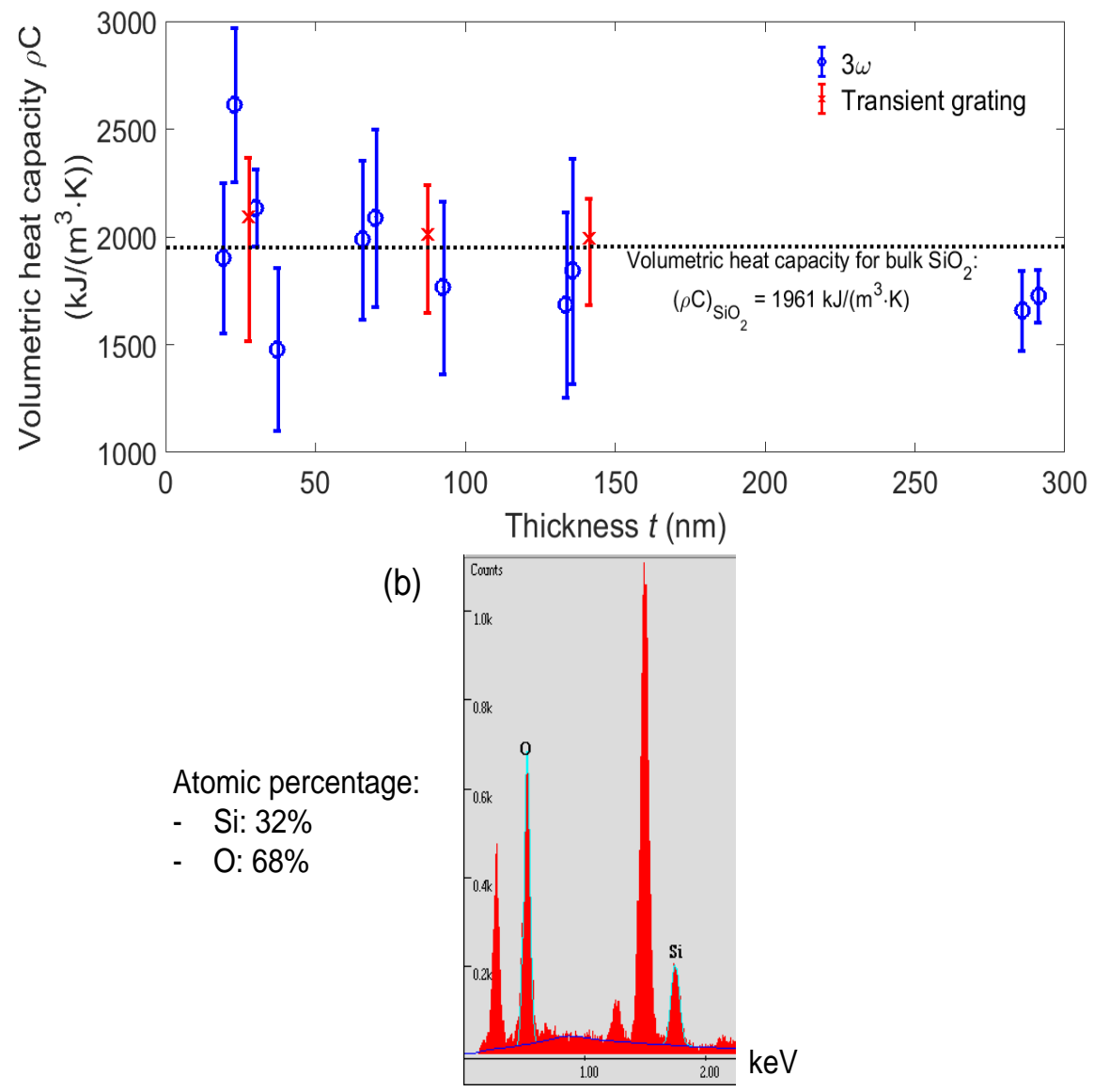

Supplementary Figure S5: (a) Plot of the volumetric heat capacity as a function of the thickness. The volumetric heat capacity has been computed from the thermal conductivity and the thermal diffusivity results presented in the Figures 3 and 4 of the main part of the article. The black dotted line corresponds to the bulk value of amorphous silica volumetric heat capacity. ${ }^{8}$ (b) EDS spectrum of a $37 \mathrm{~nm}$ thick suspended silica membrane. The atomic percentage of the elements inside the thin film can be retrieved from this spectrum. We used a $5 \mathrm{keV}$ accelerating voltage of the incident electron beam to measure the atomic percentage of an area of $90 \mu \mathrm{m} \times 90 \mu \mathrm{m}$ of the surface of the film. This result shows that in this case that the $\mathrm{SiO}_{2}$ film is almost pure, however this result depends on the samples (see Table S1). 
chamber before each measurement. The electrical resistances of the Al wires show a linear behaviour even for temperatures higher than $400 \mathrm{~K}$ as the figure $\mathrm{S} 6$ shows. The possibility of modification of the results due to $\mathrm{Al}$ non-linear TCR is limited due to three reasons: the annealing of the samples at $673 \mathrm{~K}$ for 30 minutes during the microfabrication process, the majority of the measurements has been carried out for a temperature lower than $400 \mathrm{~K}$ where the TCR of $\mathrm{Al}$ is linear ${ }^{9}$ and we included the uncertainty of TCR measurements in the final uncertainty of the presented results.

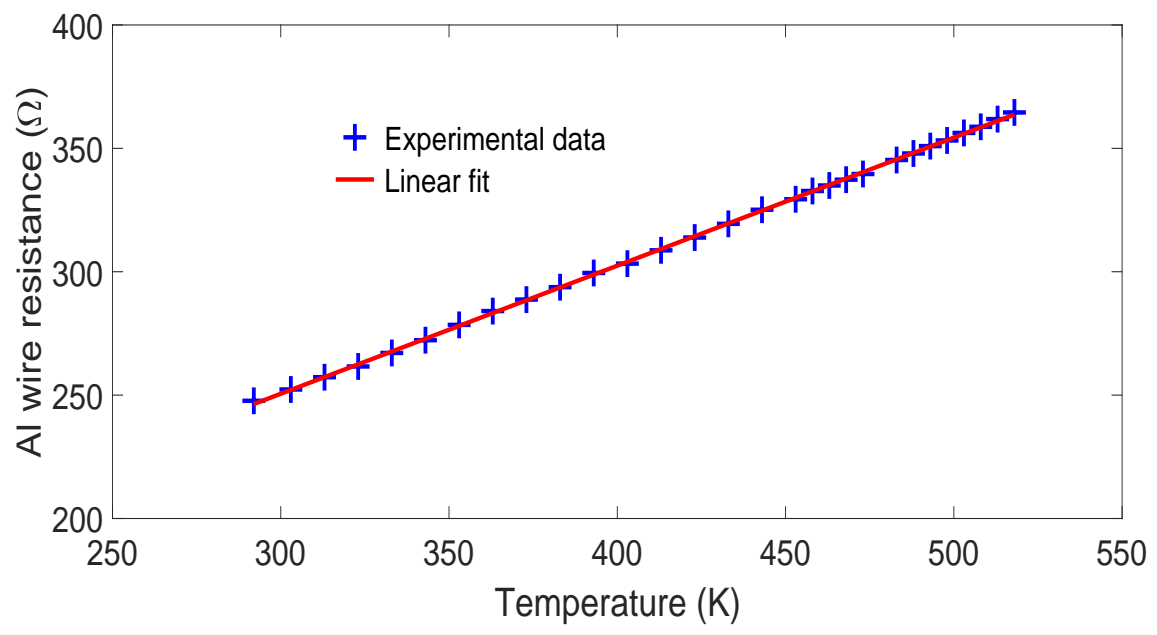

Supplementary Figure S6: Electrical resistance of Al strip wire at the measurement temperature of a $25 \mathrm{~nm} \mathrm{SiO}_{2}$ film.

After the TCR calibration the samples are placed in a vacuum chamber and are electrically connected with wires soldered with silver paste on the aluminum pads. Two connections with different aluminum pads on each side of the membrane will be used for current and voltage measurements separately. The vacuum chamber is connected to a pump, capable of delivering a pressure lower than $10^{-2} \mathrm{~Pa}$ allowing to suppress the convective heat transfer. Concerning the radiative losses even if the membranes have a very large surface, their thickness is smaller 
than the radiative penetration depth making it transparent in the infrared. To prove it, we computed the absorptivity for a $300 \mathrm{~nm}$ thick glass membrane in the infrared range where the radiative heat transfer is the strongest at room temperature (from $5 \mu \mathrm{m}$ to $80 \mu \mathrm{m}$ ) showing an average absorptivity of 0.09 (Figure S7). However if the surface of the film is too large (width larger than $500 \mu \mathrm{m}$ ) radiation losses cannot be neglected. Nevertheless the thermal model can still be used without modifications for a reasonable heater temperature rise (see the subsection "Study of the radiation losses"). Measurements are carried out at room temperature. A Tektronix AFG3022C function generator supplies DC and AC voltage for heating the samples. We measured the electrical current with an Advantest R6581 digital multimeter and the DC voltage across the metal heater with a Yokogawa 7555 digital multimeter.

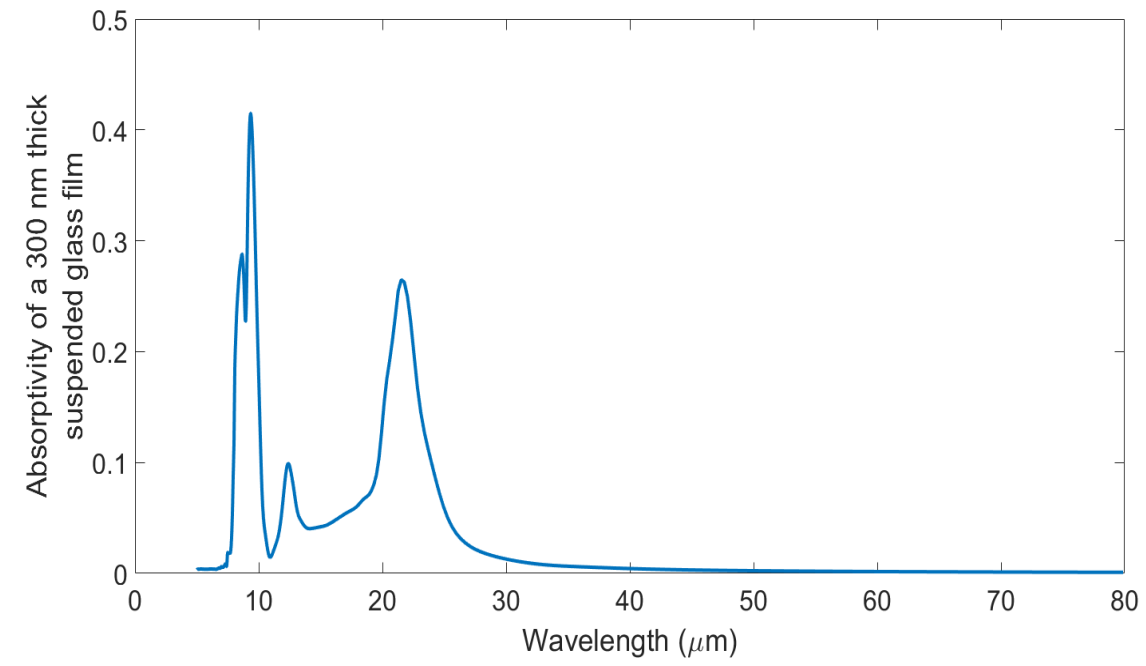

Supplementary Figure S7: Absorptivity of a $300 \mathrm{~nm}$ thick suspended silica membrane as a function of the wavelength. The absorptivity does not exceed 0.1 for most mid-infrared wavelengths.

We developed a Labview program to obtain the voltage harmonics when an AC voltage is applied to the aluminum wire. In this case voltage and current measurements are digitalized 
thanks to a data acquisition system from National Instruments. The voltage across the sample and across a reference resistance in series are measured. The voltage across the reference resistance has only an $\omega$ component which is substracted after appropriate amplification to the voltage across the metal heater. By this method we can retrieve the $3 \omega$ component of the voltage across the sample.

The principle of the thermal conductivity and thermal diffusivity measurement is exactly the same as the one proposed by Jain and Goodson ${ }^{4}$ thus it will be briefly summarized here. The electrical resistance of the metal wire is measured as a function of the temperature (Figure S8a) to obtain its temperature coefficient $\frac{d R}{d T}$. After this the DC voltage measurements will allow us to obtain the heater temperature rise $\bar{\theta}_{D C}$ through the formula $V_{D C}=R_{0} I_{0}+I_{0} \frac{d R}{d T} \bar{\theta}_{D C}$ with $R_{0}$ the resistance of the sample without heating and $I_{0}$ the current amplitude. The plot of this temperature as a function of the input electrical power enables to determine the in-plane thermal conductivity using equation (13) (Figure S8b). Next by using our Labview program we determine the $3 \omega$ component of the voltage which

can be linked to the AC temperature rise $\bar{\theta}_{A C}$ by $V_{3 \omega}=\frac{I_{0} \frac{d R}{d T} \bar{\theta}_{A C}}{2}$. With the previous measurement of the in-plane thermal conductivity we use a least-mean square fit of the experimental data with the equation (20) to retrieve the thermal diffusivity (Figure S9).

\section{Heating of the samples}

We also carried out a measurement of the thermal conductivity of the samples for different temperatures. The experimental setup is presented in the Figure S10. The sample is deposited on an aluminum plate in which a thermocouple is embedded. A ceramic heater, designed by the Sakaguchi Electric Heaters Corporation, is located below the aluminum plate and will heat the whole structure. The temperature is controlled by a proportionnal-integralderivative (PID) controller SR253 provided by Shimaden. Some grease is added between the electric heater and the aluminum plate to reduce the thermal resistance. All these measure- 

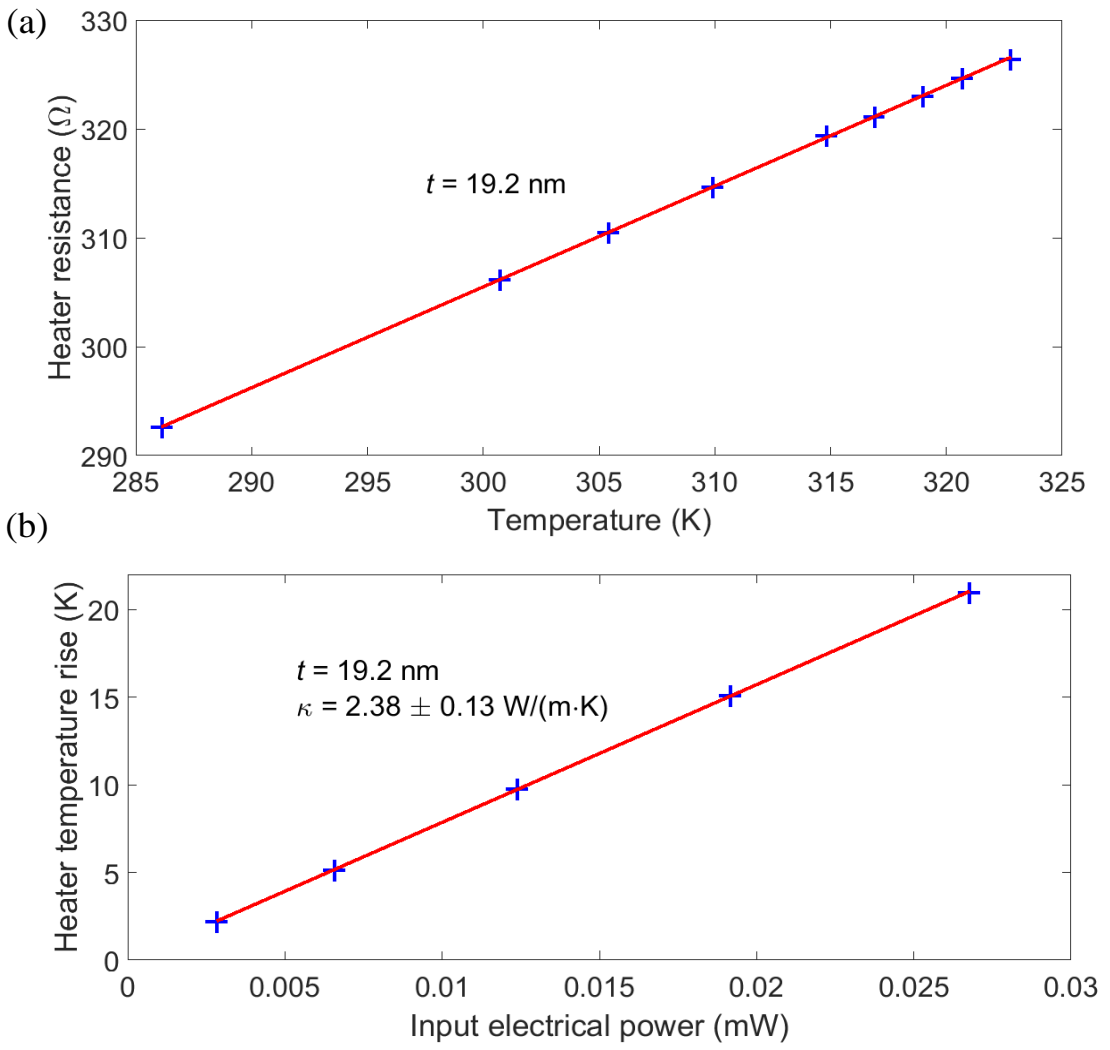

Supplementary Figure S8: (a) Plot of the heater electrical resistance of the aluminum wire deposited on a $19.2 \mathrm{~nm}$ thick silica film as a function of the temperature. We observe a good linear temperature dependence of the aluminum wire. (b) Plot of the heater temperature rise as a function of the input heating electrical power for a $19.2 \mathrm{~nm}$ thick silica film. The agreement between the linear fit and the experimental data is good and enables us to determine the thermal conductivity of the sample. 


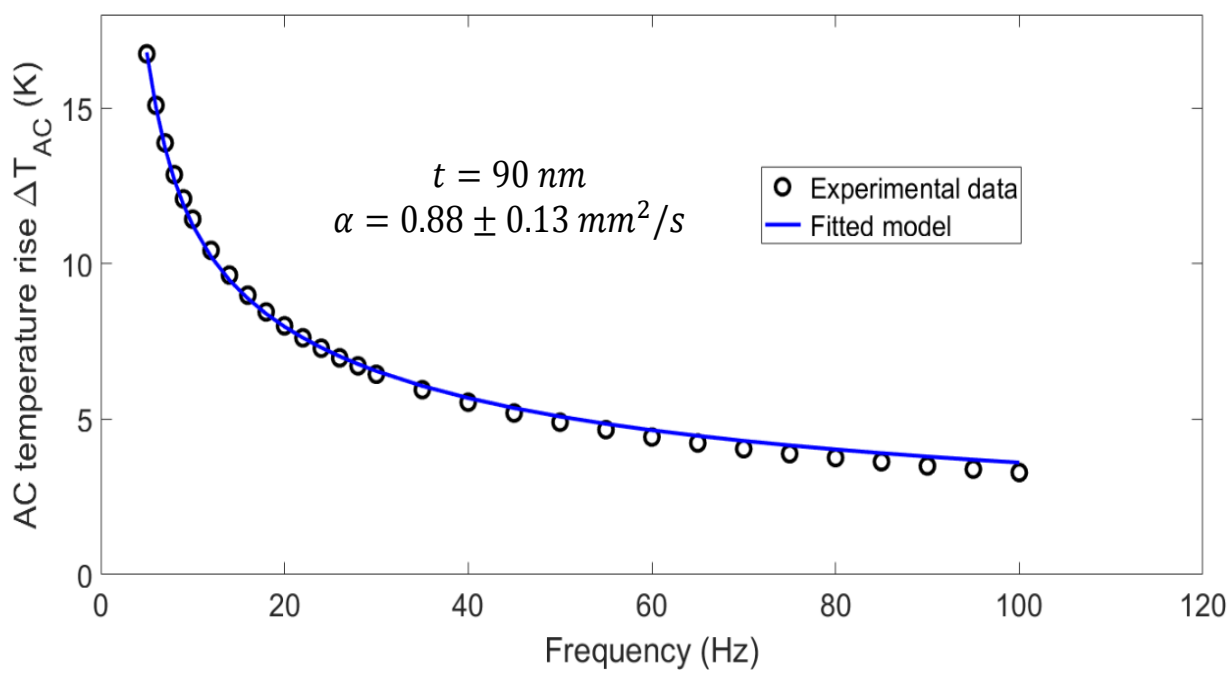

Supplementary Figure S9: Plot of the $2 \omega$ temperature oscillations as a function of the heating current frequency for a $90 \mathrm{~nm}$ thick film. There is a good agreement between the experimental and the fitted model. This fit enables us to determine the thermal diffusivity of the suspended membranes.

ments have been made under vacuum so as the measurements at room temperature.

Four samples with different thicknesses $(25 \mathrm{~nm}, 30 \mathrm{~nm}, 45 \mathrm{~nm}$ and $211 \mathrm{~nm}$ ) have been measured for temperatures between $290 \mathrm{~K}$ and $520 \mathrm{~K}$. The results are presented in the Figure $3 \mathrm{c}$ in the letter and in the Figure S11. The 25 and $30 \mathrm{~nm}$ thick samples reveal an increase of the thermal conductivity similar to the data obtained for bulk silicon dioxide ${ }^{10}$ (Figure S11a and b) and showing no evidence of enhancement due to surface electromagnetic waves. We think that the purity and the wrinkles of the samples may strongly affect the transport of the surface waves in this case. However the $211 \mathrm{~nm}$ thick sample shows that the contribution of the surface electromagnetic to the thermal conductivity will be weak for all the temperatures considered as predicted theoretically by the models I and II (Figure S11c). 


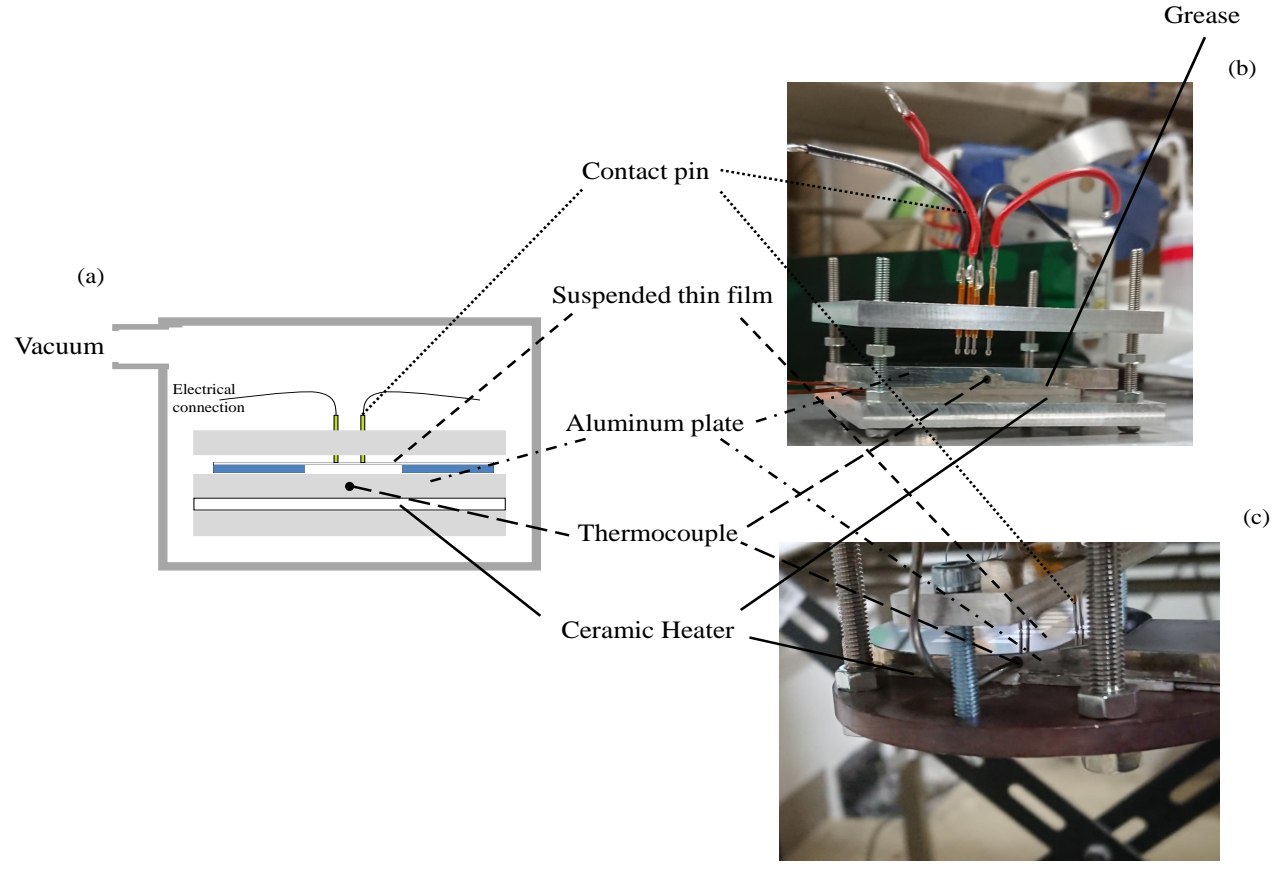

Supplementary Figure S10: Experimental setup to measure the thermal conductivity while heating the sample: (a) scheme of the setup, (b) side view picture, (c) top view picture.

\section{Transient grating technique}

The Laser-induced transient grating $(\mathrm{TG}) \operatorname{method}^{11,12}$ is a time resolved technique in which two short laser pulses are crossed at an angle $2 \theta^{\prime}$ in order to produce an intensity pattern with a periodicity $\Gamma=\lambda /\left(2 \sin \theta^{\prime}\right)$, where $\lambda$ is the optical wavelength. Absorption of the laser light by the sample creates a spatially periodic temperature profile, which will remain until the thermal energy is redistributed from peak to null. This temperature decay will induce temperature dependent changes in the refractive index that can be monitored via the diffraction of a continuous wave (CW) laser. Figure S12 shows a schematic of our TG setup, where we have implemented a heterodyne detection scheme. We refer the reader to the references ${ }^{13,14}$ for extensive details. Briefly, a transmission optical diffraction grating (phase mask) is used to split the excitation and the probe beams into two pairs. A two lens confocal imaging system is used to recombine the excitation and probe beams onto the 
(a)

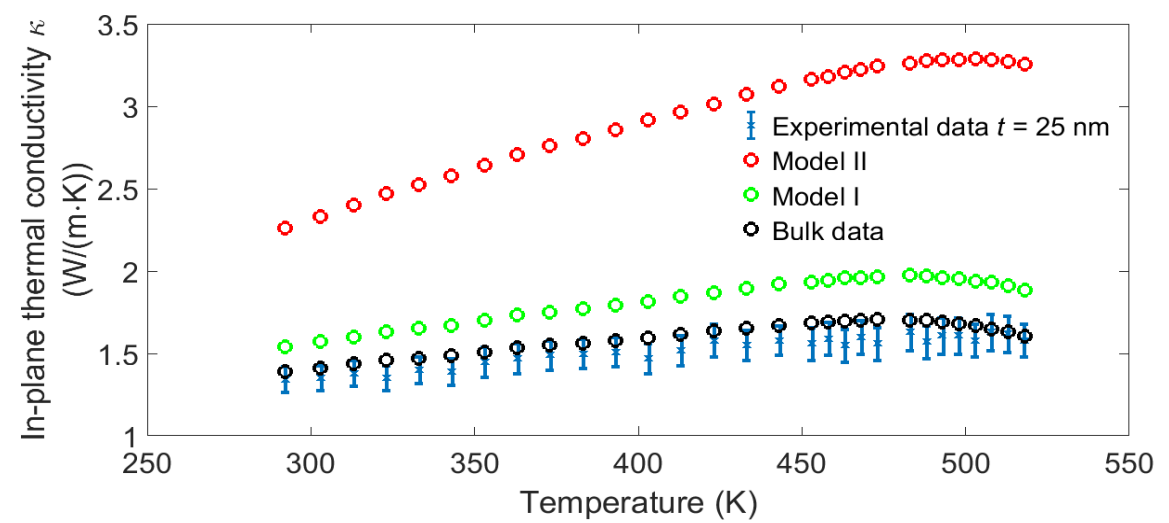

(b)

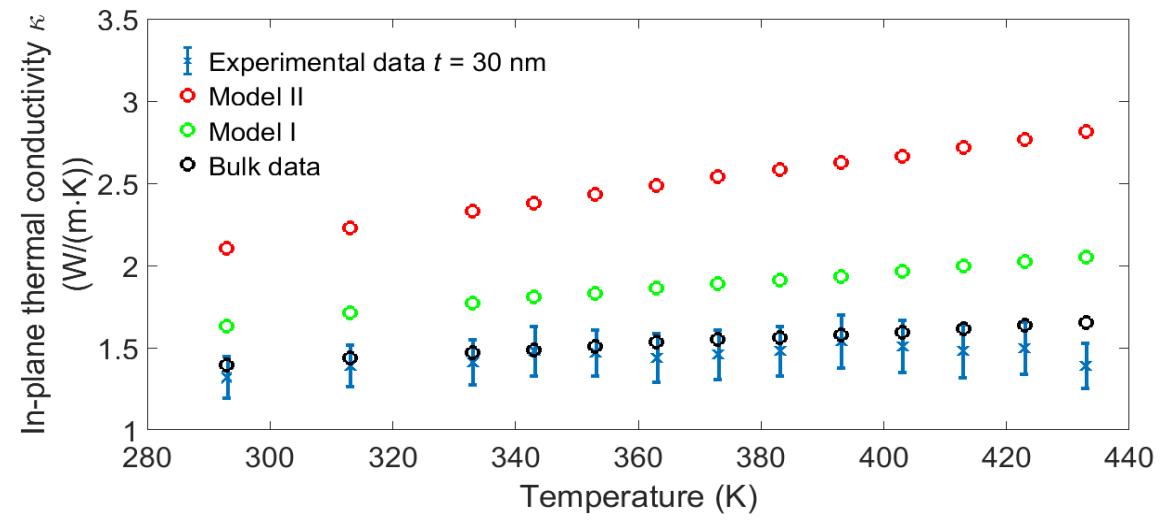

(c)

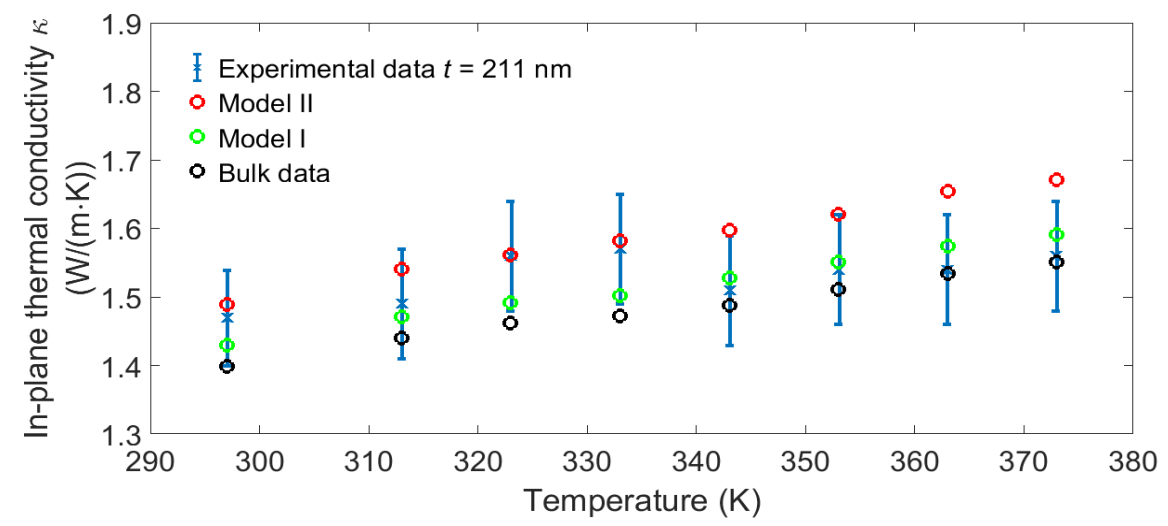

Supplementary Figure S11: Plot of the in-plane thermal conductivity as a function of the film temperature for three different samples. The experimental data are compared with previous measurements of bulk silicon dioxide as a function of the temperature ${ }^{10}$ and with the same data to which are added the predictions of model I and II. 
sample ( $5 \mathrm{~cm}$ and $15 \mathrm{~cm}$ focal length). One of the probe beams is attenuated and used as the local oscillator (reference). The beam overlap of the reference and the diffracted probe light leads to amplification and linearization of the observed signal (heterodyne detection), ${ }^{13,15}$ and is subsequently monitored using a Thorlabs PD100A photodiode connected to a Tektronix DPO7000 oscilloscope.

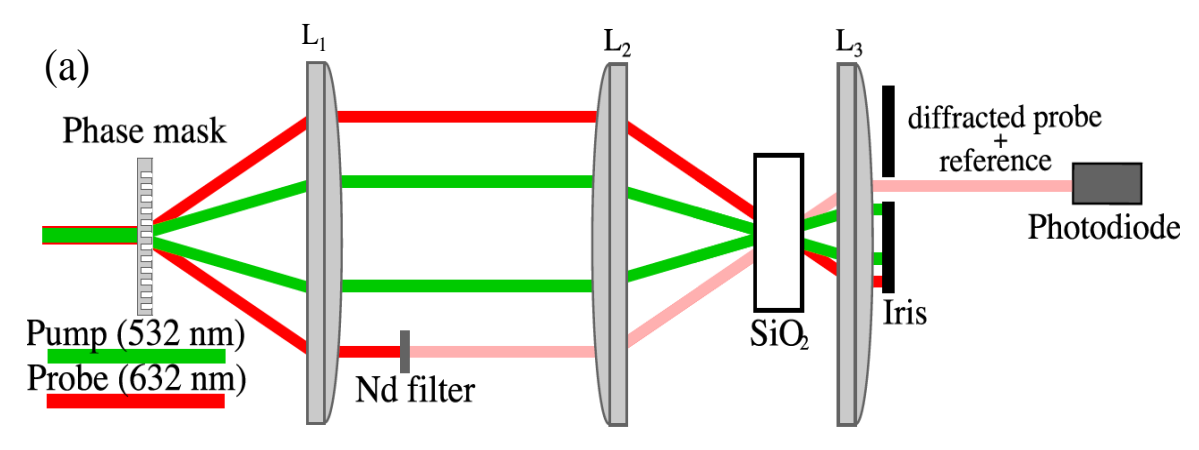

(b)
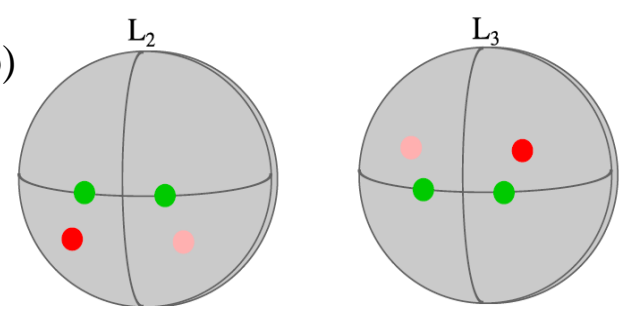

Supplementary Figure S12: Top-down view of the Transient grating (TG) setup with optical heterodyne detection for $\mathrm{SiO}_{2}$ samples. Both beams, the pulsed pump and CW probe, are collinear before going into the TG setup. They are both focused onto a same point on a transmission diffraction grating, which splits the beams into multiple diffraction orders. All the diffracted orders are blocked except for 1 order. Lens L1 (focal distance of $7.5 \mathrm{~cm}$ ) collimates the beams and lens L2 (focal distance of $15 \mathrm{~cm}$ ) focuses the beams onto the sample. Lens L3 (focal distance of $7.5 \mathrm{~cm}$ ) is used in order to focus the diffracted probe plus reference beam into the detector. (b) Front view of lenses L2 and L3 as observed from left to right in (a). The probe beam-pair has a vertical offset with respect to the excitation in order to allow heterodyne detection.

The excitation pulses (pump beams) have $8 \mathrm{~ns}$ pulse width, $20 \mathrm{~Hz}$ repetition rate and are frequency doubled to $532 \mathrm{~nm}$ wavelength. The spot diameter at the sample was $350 \mu \mathrm{m}$ with $\mathrm{a} \sim 100$ pulse energy. The probe beam is a CW He-Ne laser with $632 \mathrm{~nm}$ wavelength, 270 $\mu \mathrm{m}$ spot diameter and $7.6 \mathrm{~mW}$ power. In semitransparent samples, heat diffusion induced by the TG technique is one-dimensional if the crossing depth of the excitation pulses is much 
larger than the grating period (this condition is satisfied for the $\mathrm{SiO}_{2}$ membranes). In this case the temperature evolution can be expressed as a decaying exponential: ${ }^{11}$

$$
T(x, t)=T_{0} e^{-\frac{t}{\tau}}, \quad \tau=\frac{\Gamma^{2}}{4 \pi^{2} \alpha}
$$

where $\Gamma$ is the TG periodicity and $\alpha$ is the thermal diffusivity of the film. Figure S13 shows a typical time trace $(\Gamma=32 \mu \mathrm{m})$ obtained along with the best exponential fit (dashed line) for a $\mathrm{SiO}_{2}$ membrane of thickness $\approx 140 \mathrm{~nm}$. Since the $\mathrm{SiO}_{2}$ is optically transparent to the excitation wavelength, $\mathrm{a} \sim 2.8 \mathrm{~nm}$ layer of $\mathrm{AuPd}$ was deposited on the film in order to produce the temperature grating. Three different wafers were measured. Each wafer had 7 membranes of the same thickness. In total 3 different membrane thicknesses were measured (141.2 nm, $87.3 \mathrm{~nm}$ and $27.5 \mathrm{~nm})$, and three different grating periods were employed for each membrane (30, 32 and $36 \mu \mathrm{m})$. Given the spot size of the probe beam and the grating period, the TG decay represents the average diffusion of heat across 7-9 grating periods.

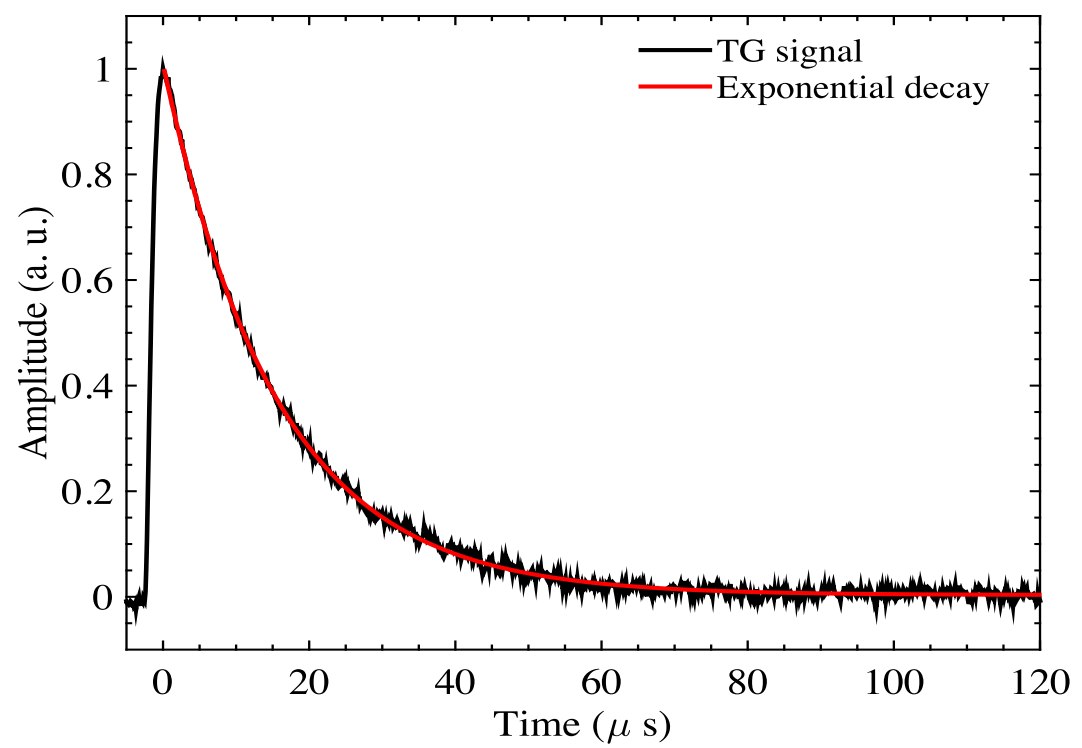

Supplementary Figure S13: Typical TG signal obtained for a $\mathrm{SiO}_{2}$ membrane of $140 \mathrm{~nm}$ thickness using a grating period of $32 \mu \mathrm{m}$. Solid red line corresponds to the best fit using equation (25). 
Figure $\mathrm{S} 14$ shows the thermal diffusivity of the measured $\mathrm{SiO}_{2}$ membranes as a function of the membrane thickness. The error bars are the standard deviation between the measured grating periods. In addition, the effect that the AuPd film has on the measured thermal properties has to be also taken into account. This is because the TG experiment measures the "effective" thermal diffusivity of the $\mathrm{SiO}_{2} / \mathrm{AuPd}$ two layer system. The actual value of the thermal diffusivity of $\mathrm{SiO}_{2}$ will be lower compared to the measured effective value. The precise determination of the $\mathrm{SiO}_{2}$ diffusivity without the AuPd film would require exact information regarding thermal boundary resistance between layers and electron mean free path of AuPd. However, the effect will be small due to the thickness of the AuPd film being much smaller than the $\mathrm{SiO}_{2}$ membrane. In this case, as a first approximation, we consider that the thermal diffusivity measured with the TG experiment corresponds to the thermal diffusivity of the $\mathrm{SiO}_{2}$ membrane and the effect of the $\mathrm{AuPd}$ film is to increase the error bars towards lower values (asymmetrical error bar shown in Figure S14).

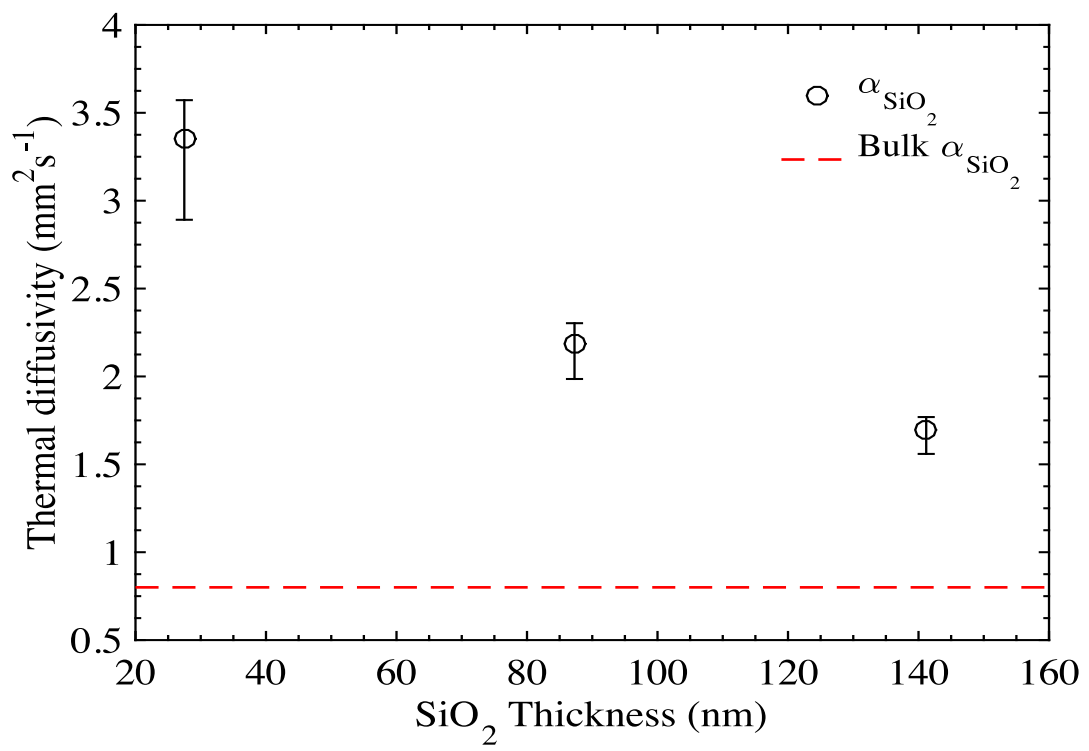

Supplementary Figure S14: Thermal diffusivity as a function of the $\mathrm{SiO}_{2}$ film thickness. 
The thermal conductivity of the $\mathrm{SiO}_{2}$ membranes is shown in Figure $\mathrm{S} 15$ and can be calculated using the following expression:

$$
\kappa_{\mathrm{SiO}_{2}}=\alpha_{\mathrm{SiO}_{2}}(\rho C)_{e f f}
$$

where

$$
(\rho C)_{e f f}=\frac{L_{\mathrm{SiO}_{2}}}{L_{T}}(\rho C)_{\mathrm{SiO}_{2}}+\frac{L_{\mathrm{AuPd}}}{L_{T}}(\rho C)_{\mathrm{AuPd}}
$$

$\alpha_{\mathrm{SiO}_{2}}$ is the measured thermal diffusivity (see Figure S14), $L_{T}=L_{\mathrm{AuPd}}+L_{\mathrm{SiO}_{2}}, L_{\mathrm{AuPd}}$ is the thickness of the AuPd layer and $L_{\mathrm{SiO}_{2}}$ is the thickness of the $\mathrm{SiO}_{2}$ membrane. The volumetric heat capacity of $\mathrm{AuPd}$ is $(\rho C)_{A u P d}=3393.4 \mathrm{~kJ} /\left(\mathrm{m}^{3} \cdot \mathrm{K}\right)^{16}$ and the volumetric heat capacity of $\mathrm{SiO}_{2}$ is $(\rho C)_{\mathrm{SiO}_{2}}=1961 \mathrm{~kJ} /\left(\mathrm{m}^{3} \cdot \mathrm{K}\right) .{ }^{8}$ The density of the AuPd alloy $\rho_{A u P d}$ was calculated by averaging the density of $\mathrm{Au}$ and $\mathrm{Pd}$ weighted by the stoichiometric composition ( $80 \% \mathrm{Au}$ and $20 \% \mathrm{Pd})$.

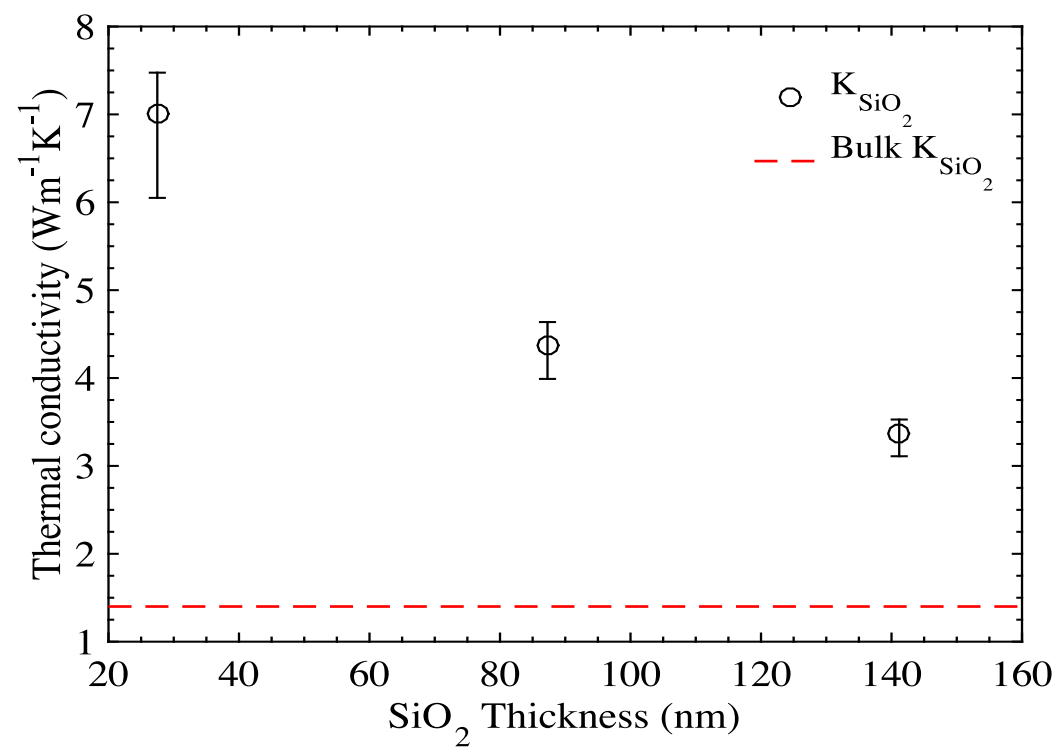

Supplementary Figure S15: Thermal conductivity as a function of the $\mathrm{SiO}_{2}$ film thickness.

We also present the results obtained for each individual grating, Figure S16a shows the thermal conductivity and Figure S16b the thermal diffusivity of the silica membrane as a 
function of the grating period. The thermal conductivity and thermal diffusivity increase as the thickness reduces regardless of the grating period. 
(a)

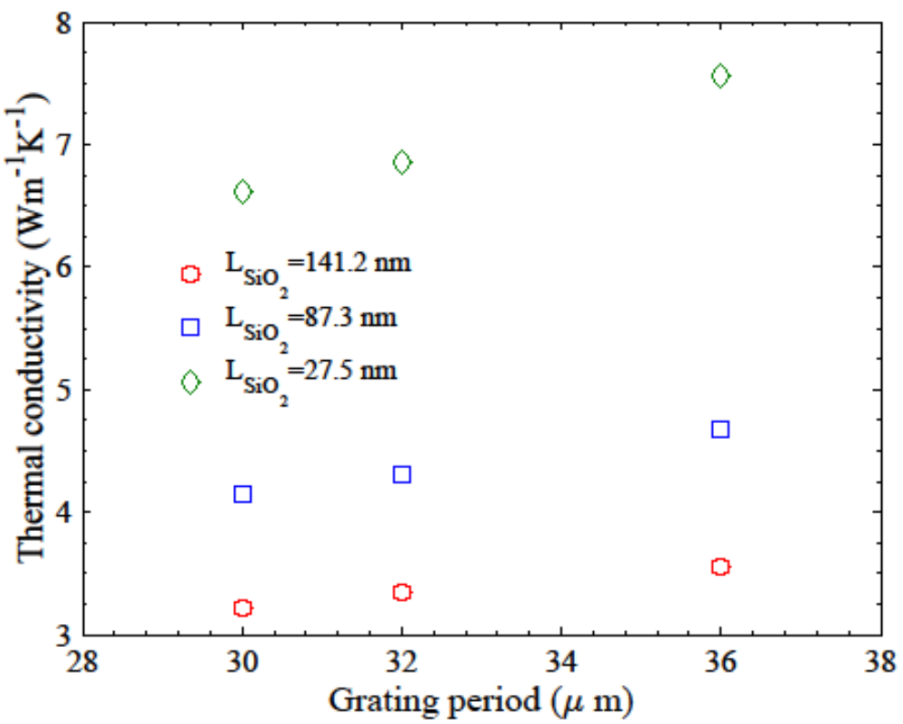

(b)

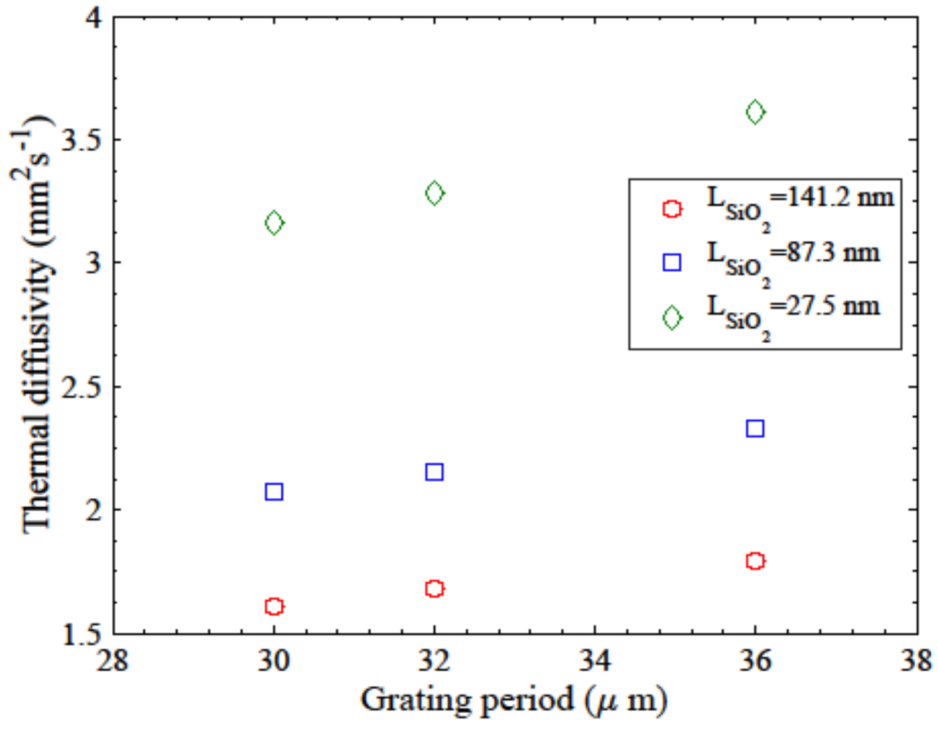

Supplementary Figure S16: (a) Thermal conductivity and (b) thermal diffusivity as a function of the grating period. 


\section{Calculation of the effect of the nanometer AuPd film on the thermal measurements}

In order to calculate the error associated with the presence of the AuPd film we first have to calculate the thermal conductivity of the film $\kappa_{A u P d}$. Given the nanometer thickness of the film $(2.8 \mathrm{~nm})$, the thermal conductivity will be reduced compared to the bulk value due to boundary effects. This reduction can be calculated using the following equation: ${ }^{17}$

$$
\frac{\kappa_{A u P d}^{f i l m}}{\kappa_{A u P d}^{b u l k}}=1-\frac{3}{8 x}\left(1-4 E_{3}(x)+4 E_{5}(x)\right),
$$

where

$$
E_{n}(x)=\int_{0}^{1} \mu^{n-2} \exp ^{-x / \mu} d \mu, \quad x=\Lambda_{A u P d} / L_{A u P d},
$$

$\Lambda_{A u P d}$ is the electron mean free path (MFP), $L_{A u P d}$ is the film thickness and $\kappa_{A u P d}^{b u l k}=54.9$ $\mathrm{W} /(\mathrm{m} \cdot \mathrm{K})$ is the bulk thermal conductivity of the AuPd alloy (Au 80\%, Pd 20\%). ${ }^{18}$ The MFP of the AuPd alloy has been estimated by using Matthiessen's rule and the reported MFPs for Au and for Pd:

$$
\frac{1}{\Lambda_{A u P d}}=\frac{1}{\Lambda_{A u}}+\frac{1}{\Lambda_{P d}},
$$

where $\Lambda_{A u}=41.7 \mathrm{~nm}^{19}$ and $\Lambda_{P d}=22.5 \mathrm{~nm} .{ }^{20}$ Substituting these values into equations (28) and (30), the thermal conductivity of the AuPd film is $\kappa_{A u P d}^{f i l m} \approx 16.5 \mathrm{~W} /(\mathrm{m} \cdot \mathrm{K})$. The in-plane effective thermal conductivity for two layers can be expressed using the following equation:

$$
\kappa_{e f f}=\frac{L_{A u P d}}{L_{T}} \kappa_{A u P d}^{f i l m}+\frac{L_{S_{i O}}}{L_{T}} \kappa_{S i O_{2}}^{f i l m},
$$

with this, we can calculate the lower-limit value of the $\mathrm{SiO}_{2}$ thermal conductivity:

$$
\kappa_{\mathrm{SiO}_{2}}^{f i l m}=\frac{L_{T}}{L_{\mathrm{SiO}_{2}}} \kappa_{e f f}-\frac{L_{\mathrm{AuPd}}}{L_{\mathrm{SiO}_{2}}} \kappa_{\mathrm{AuPd}}^{f i l m} .
$$

This lower-limit is taken as the lower-bounds of the error bars. 


\section{References}

(1) Ordonez-Miranda, J.; Tranchant, L.; Tokunaga, T.; Kim, B.; Palpant, B.; Chalopin, Y.; Antoni, T.; Volz, S. Anomalous Thermal Conductivity by Surface Phonon-Polaritons of Polar Nano Thin Films due to their Asymmetric Surrounding Media. J. Appl. Phys. 2013, 113, 084311-084318.

(2) Chen, D.-Z.; Narayanaswamy, A.; Chen, G. Surface Phonon-Polariton Mediated Thermal Conductivity Enhancement of Amorphous Thin Films. Phys. Rev. B: Condens. Matter Mater. Phys. 2005, 72, 155435-155438.

(3) Gluchko, S.; Palpant, B.; Volz, S.; Braive, R.; Antoni, T. Thermal Excitation of Broadband and Long-Range Surface Waves on $\mathrm{SiO}_{2}$ Submicron Films. Appl. Phys. Lett. 2017, 110, 263108-263111.

(4) Jain, A.; Goodson, K. E. Measurement of the Thermal Conductivity and Heat Capacity of Freestanding Shape Memory Thin Films Using the $3 \omega$ Method. J. Heat Transfer 2008, 130, 102402-102408.

(5) Senturia, S. D. Microsystem Design; Kluwer Academic Publishers, 2001.

(6) Pop, E. Energy Dissipation and Transport in Nanoscale Devices. Nano Res. 2010, 3, $147-169$.

(7) Chalopin, Y.; Hayoun, M.; Volz, S.; Dammak, H. Surface Enhanced Infrared Absorption in Dielectric Thin Films with Ultra-Strong Confinement Effects. Appl. Phys. Lett. 2014, 104, 011905-011908.

(8) Haynes, W. M. CRC Handbook of Chemistry and Physics; CRC Press: Boca Raton, FL, 2006.

(9) Dames, C. Measuring the Thermal Conductivity of Thin Films: 3 Omega and Related Electrothermal Methods. Annu. Rev. Heat Transfer 2013, 16. 
(10) Zeller, R. C.; Pohl, R. O. Thermal Conductivity and Specific Heat of Noncrystalline Solids. Phys. Rev. B 1971, 4, 2029-2041.

(11) Eichler, H. J.; Günter, P.; Pohl, D. W. Laser-Induced Dynamic Gratings; Springer: Berlin, 1986.

(12) Rogers, J. A.; Maznev, A. A.; Banet, M. J.; Nelson, K. A. Optical Generation and Characterization of Acoustic Waves in Thin Films: Fundamentals and Applications. Annu. Rev. Mater. Sci. 2000, 30, 117-157.

(13) Maznev, A.; Nelson, K.; Rogers, J. Optical Heterodyne Detection of Laser-Induced Gratings. Opt. Lett. 1998, 23, 1319-1321.

(14) Vega-Flick, A.; Eliason, J.; Maznev, A.; Khanolkar, A.; Abi Ghanem, M.; Boechler, N.; Alvarado-Gil, J.; Nelson, K. Laser-Induced Transient Grating Setup with Continuously Tunable Period. Rev. Sci. Instrum. 2015, 86, 123101-123104.

(15) Goodno, G. D.; Dadusc, G.; Miller, R. D. Ultrafast Heterodyne-Detected TransientGrating Spectroscopy Using Diffractive Optics. J. Opt. Soc. Am. B 1998, 15, 17911794.

(16) Asaoka, K. Thermal Diffusivity and Specific Heat of Dental Casting Alloys at Room and Elevated Temperatures. Dent. Mater. J. 1997, 16, 78-86.

(17) Chen, G. Nanoscale Energy Transport and Conversion: a Parallel Treatment of Electrons, Molecules, Phonons, and Photons; Oxford University Press, 2005.

(18) Ho, C.; Ackerman, M.; Wu, K.; Oh, S.; Havill, T. Thermal Conductivity of Ten Selected Binary Alloy Systems. J. Phys. Chem. Ref. Data 1978, 7, 959-1178.

(19) Omar, M. A. Elementary Solid State Physics: Principles and Applications; Pearson Education India, 1975. 
(20) Shivaprasad, S.; Udachan, L.; Angadi, M. Electrical Resistivity of Thin Palladium Films. Phys. Lett. A 1980, 78, 187-188. 